\title{
ADAPTIVE REDUCED PATHS SUCCESSIVE CANCELLATION LIST DECODING FOR POLAR CODES
}

\author{
Walled Abdulwahab ${ }^{1}$, Abdulkareem A. Kadhim² \\ ${ }^{1,2}$ College of Information Engineering, Al-Nahrain University, Baghdad, Iraq \\ $\left\{\right.$ walled.khalid ${ }^{1}$, abdulkareem. $\left.\mathrm{a}^{2}\right\} @$ coie-nahrain.edu.iq \\ Received: 12/11/2021 , Accepted: 28/01/2021
}

\begin{abstract}
Adaptive Reduced Path Successive Cancellation List (ARP-SCL) decoding for polar codes is proposed in this paper. The aim here is to reduce the complexity of the conventional Successive Cancellation List (SCL) and a previously proposed Reduced Path SCL (RP-SCL) decoders. The complexity reduction is obtained at low and moderate signal to noise power ratio (SNR) regions. The operation of the proposed ARP-SCL decoder relied on the calculation of different optimum pruning parameters according to the operating SNR, while RP-SCL used single parameters set. Simulation tests are carried out to determine both the complexity and Bit Error Rate (BER) performances of the proposed ARP-SCL decoder in comparison with SCL and RP-SCL decoders. Additive White Gaussian Noise (AWGN) channel and two fading channel models simulating indoor 5G environment using millimetre Wave (mmWave) with a carrier frequency of $28 \mathrm{GHz}$ and having either 50 or $400 \mathrm{MHz}$ bandwidths are used. The results revealed that the proposed ARP-SCL decoder reduced the average processed paths up to $46.88 \%$. The largest complexity reductions are obtained in the case of fading channel having $400 \mathrm{MHz}$ bandwidth at high SNR regions. In most test conditions, the mentioned reduction in complexity is achieved without greatly sacrificing the required BER. .
\end{abstract}

\section{INTRODUCTION}

Polar codes are linear block codes introduced in 2009 by Erdal Arikan [1]. Channel polarization is used in polar codes to realize the channel capacity of binary-input discrete memoryless channels (B-DMC). Successive cancellation (SC) is used for the decoding of polar codes. For long block length $(\mathrm{N})$, this approach achieved acceptable performance with $\mathrm{O}$ $(\mathrm{N} \cdot \log 2 \mathrm{~N})$ complexity [2,3].

Successive cancellation list (SCL) decoding was proposed with cyclic redundancy check (CRC) to promote the code performance at both low and moderate coding lengths at the expense of increased decoding complexity to O (L.N.log2 $\mathrm{N}$ ), where $\mathrm{L}$ is the list size of the decoder [2,3]. Both decoding approaches use the same initial steps, but they use a different mechanism to decode the data bits. Two decoding paths are formed with values of "0" and "1" in SCL instead of deciding the decoded data bit based on thresholding as in SC. Thus, the number of paths is duplicated for each decoding bit. Polar codes with SCL-CRC contending with other modern channel coding schemes such as low-density parity-check (LDPC) code and turbo code [4-7]. Enhanced Mobile Broadband (eMBB) is a 5G use case that used polar codes with an SCL-CRC decoder to encode the control information. Polar code is a candidate also for other 5G use cases [8,9].

Many works introduced different approaches to reduce the SCL decoding complexity. One of these approaches is decoding tree pruning [10], where any decoding path with a metric that does not satisfy a predetermined thresholding condition should be discarded. Path metric is determined based on the Log-Likelihood Ratio (LLR) of a given path at a specific level [11].

A threshold value is determined at each decoding level using the maximum metric at that level. Such a pruning approach is conservative and operated successfully at a high signal to noise power ratio (SNR) only. Such a problem was solved 
by [12], where a record of size $\mathrm{L}$ is used to define the threshold value. The record is updated at each decoding level. This record contains the removed paths which have the larger predestined performance loss. The approach reduced the complexity of the decoder at low and moderate SNR regions but at the cost of adding overhead to the decoding process. In another approach to decrease the complexity of the SCL decoder, the memory requirement is reduced by the so-called Partitioning SCL (PSCL) algorithm by breaking down the decoding tree into few decoding parts with only one candidate path being moved to the next decoding step [13]. As a consequence, the same memory space can be shared among the different decoding parts. The improvements provided by the PSCL paradigm was proved [14]. This covered the interpolation construction of polar code and the assignment of CRC with a specific length to each partition. A theoretical upper bound of SCL size was proved also. A theoretical analysis of PSCL showed the existence of a gap in error correction performance between SCL and PSCL [15].

This gap was removed by introducing generalized PSCL resulted in a lower limit for the list size and improved performance over binary eraser channel.Redundant calculations in the SCL decoder are removed in an attempt to reduce the decoder complexity [16]. This resulted in a simplified SCL decoding with performance almost equivelant to that of conventional SCL. Another fast and simple SCL decoder was suggested based on Rate-1 node, a special node used in both conventional and simplified SCL decoders [17]. Rate-1 nodes representing the decoding tree comprising information bits only. The proposed approach reduced the required time for decoding without significantly affecting the error performance. A generalized approach was devised in [18] to decrease the decoding latency of the SCL decoder by recognizing specific sub-codes inside the polar code. This approach achieved gains in term of decoding latency of about $23 \%$ and $29 \%$ compared to SC and SCL, respectively, without sacrificing the code performance.

Tree pruning approaches based on SCL path metric thresholding are already tackled by different researchers. The threshold value is updated at each decoding level, an overhead is added for the decoding process [10,12]. In these works, the performance of the proposed approaches was evaluated over additive white Gaussian noise (AWGN) channel only. A Reduced Paths Successive SCL (RP-SCL) decoder considering three different thresholds for tree pruning are suggested in [19]. These thresholds were optimized to give the least complexity and reasonable bit error rate (BER) performance for fixed SNR. The performance is evaluated in 5G environments and a relatively good complexity reduction is achieved at high SNR. In the present work, an adaptive version of RP-SCL is introduced where the thresholds are optimized according to the operating SNR region. This paper aims at obtaining better complexity reduction at low and moderate SNR regions while maintaining an acceptable BER performance of the decoder. The remaining parts of this paper are organized as follows: brief descriptions of SC, SCL, and RP-SCL are given in Section II. The operation of the proposed Adaptive Reduced Paths SCL (ARP-SCL) decoder is presented in Section III. Simulation results and discussion are presented in Section IV. Finally, the conclusion is given in Section V.

https://ijict.edu.iq 


\section{SUCCESSIVE CANCELATION BASED DECODING APPROACHES}

\section{A. Successive Cancellation Decoding}

In the early years of introducing polar codes, successive cancellation (SC) algorithm is used in the decoding [4,20]. The Binary tree is used to represent polar codes. SC utilizes this tree for decoding by picking the left edges until reaching the leaf nodes followed by backtracking to the right one. At the leaf nodes, the decoding is based on the type of the node. If the node is a frozen one then it set to zero. In the case of information nodes, threshold detection is used to determine the bit value. When a node gets the estimated values from both of its children, it combined them and pushed them to its parent node $[5,20]$.

For long block length $(\mathrm{N})$, SC achieved acceptable performance with $\mathrm{O}(\mathrm{N} \cdot \log 2 \mathrm{~N}$ ) complexity. Unfortunately, the performance of the SC decoder is not as good as that of the maximum-likelihood (ML) decoding for short and moderate block lengths. This is an important issue in 5G applications [4,5]. To overcome this weakness, and improved SC (called SC list) decoding is introduced.

\section{B. Successive Cancellation List Decoding}

A new integer parameter, list size (L) is added to the decoding process of the SC decoder. In such an approach, abbreviated as SCL, a set of SC decoders work together and maintain different candidate paths. At the leaf nodes and in the case of information nodes, both bit values 0 and 1 are considered and the number of candidate paths is duplicated [21]. This process is repeated at each information bit so that the number of decoding paths is duplicated. Path metric $\left(C_{l}^{(i)}\right)$ as given in (1) is assigned for each decoding path to specify its likelihood [11].

$$
C_{l}^{(i)} \triangleq \sum_{j=0}^{i} \ln \left(1+e^{-\left(1-2 \hat{u}_{j}[l]\right) \cdot L_{n}^{(j)}[l]}\right)
$$

Where $C_{l}^{(i)}$ is the metric value of $l^{\text {th }}$ path at $i^{\text {th }}$ level, $\hat{u}_{j}[l]$ is the decoded bit at $j^{\text {th }}$ level $(0 \leq j \leq i)$ and thh path, $n$ is the Kronecker matrix power used to produce the codeword, and $L_{n}^{(j)}[l]$ is the Log-Likelihood Ratio (LLR) for bit $u_{i}$ and defined by (2) [11]:

$$
L_{n}^{(i)}[l]=\ln \left(\frac{w_{n}^{(i)}\left(y, \hat{u}^{i-1}[l] \mid 0\right)}{w_{n}^{(i)}\left(y, \hat{u}^{i-1}[l] \mid 1\right)}\right)
$$

with $\hat{u}^{i-1}$ being the past route of the path and $w_{n}^{(i)}$, with $\mathrm{N}=2^{n}$ and $i \in \mathrm{N}$, is a discrete binary memoryless channel with $u$ and $y$ as its input and output, respectively.

In the case where the number of decoding paths exceeds $\mathrm{L}$ at a certain decoding level, a pruning process is applied based on path metrics to keep the most likely L paths only (those with the least metrics). At the end of the decoding process, a cyclic redundancy check (CRC) is used to pick up a decoding path among the most likely L paths as the output of the decoding process [11,21]. SCL decoding succeeded in improving the performance of polar codes but at the cost of increased complexity. The complexity is increased from $\mathrm{O}(\mathrm{N} \log \mathrm{N})$ for SC decoder to O(L.N .Log N) with SCL 
decoder [3,11]. For a wide range of polar code block lengths and rates, SCL bridged the gap between the performance of SC decoding and that of ML decoding [4,5].

\section{Reduced Paths Successive Cancellation List Decoding}

Reduced Paths Successive Cancellation List (RP-SCL) introduced different pruning mechanisms in the decoding of the SCL decoder to reduce the average number of the processed paths. Three thresholds were defined

for this purpose: two thresholds (called $\mathrm{T}_{\alpha}$ and $\mathrm{T}_{\gamma}$ ) are based on the path metrics at each level. These two thresholds are defined in (3) and (4), respectively [19]:

$$
\begin{gathered}
T_{\alpha}=C_{\max }-\alpha C_{\max } \quad, \quad 0<\alpha<1 \\
T_{\gamma}=\gamma \bar{C}_{l}, \quad \gamma \geq 1
\end{gathered}
$$

where $C_{\max }$ and $\bar{C}_{l}$ are, respectively, the maximum path metric and the average of metric values at given decoding level. Any path with metric exceeding these thresholds will be discarded. The threshold $\mathrm{T}_{\gamma}$ is used only in the case where $\mathrm{T}_{\alpha}$ failed in producing any decoding path due to its discarding rule. The third threshold (called $D_{\beta}$ ) is used to specify the decoding tree depth at which the pruning of RP-SCL is taken place. $D_{\beta}$ is defined in (5) [19]:

$$
D_{\beta}=\lceil\beta \cdot K\rceil, \quad 0 \leq \beta<1
$$

where $\mathrm{K}$ is the data block length (in bits) of the polar encoder and $\lceil S\rceil$ is the ceiling (upper integer) of S. Optimum values for the pruning parameters $\gamma, \alpha$, and $\beta$ mentioned in (3), (4), and (5), respectively, are used. The optimization is based on taking the parameter values that achieved the best complexity reduction in terms of the average processed paths $\left(L_{a y}\right)$ by the decoder combined with acceptable degradation in the BER performance of the decoder. $\alpha_{o p t}$ is determined first, followed by $\beta_{o p t}$ and then $\gamma_{o p t}$. A design (fixed) SNR is used in the optimization process that corresponds to BER of $10^{-4}$ of the SCL decoder. Although, such an approach produced complexity reduction with slight BER degradation, using single design SNR in the optimization process affected the achieved advantages of RP-SCL decoder at low and moderate SNR"s. This is the main reason behind the proposal of the ARP-SCL in this work, where different SNR regions are considered in the optimization as will be described in the next section.

\section{The Proposed Adaptive Reduced Paths Successive Cancelation List decoding}

Analyzing the operation of the RP-SCL decoder and the optimization of its parameters revealed that the majority of the correct paths are located at the beginning of the list. Further, considering $\alpha_{\mathrm{opt}}$ produced $L_{g \underline{v}}$, while $B_{\text {opt }}$ and Yopt provided a compromise between the decoder complexity $\left(L_{a \underline{v}}\right)$ and its BER performance [19]. This clarifies that Lay is determined based on the three mentioned optimum parameters. The number of pruned paths depends on the path metric, determined by (1), which in turns depend on both the channel $w$ and the SNR level. As a consequence, the pruning parameters are a function of the channel and the operating SNR. Thus, according to (1)- (5), one may conclude that $T_{\alpha}, T_{\psi}$, and $D_{B}$ depend on both the channel and the operating SNR. The relation between the optimum pruning parameters and the operating 
SNR just described together with the relatively mild complexity reduction of RP-SCL at low and moderate SNRs give the motivation to propose an adaptive version of RP-SCL. Flexibility is provided by ARP-SCL in determining different sets of optimum pruning parameters $\left(\alpha_{o p t}, \beta_{o p t}\right.$, and $\left.\gamma_{o p t}\right)$, each set corresponding to an operating SNR region rather than related to single (fixed) SNR as in RP-SCL. The main goal here is to improve the capability of the decoder in reducing the complexity at both low and moderate SNR regions which are important in cellular networks [12].

As in the RP-SCL decoder, the pruning parameters of ARP-SCL are determined before the actual transmission. According to the expected BER range and the corresponding SNR, the optimum pruning parameters are determined and stored in a look-up table according to the SNR regions. As the operating SNR changed during transmission, SNR level estimation is determined and passed to the decoder as an estimate for the current SNR. The optimum parameters corresponding to the estimated SNR region are used in the decoding process of the ARP-SCL decoder. The ARP-SCL decoder keeps operating with a new set of parameters until a change in estimated SNR occurred. Algorithm-I shows the processing steps of the ARP-SCL decoder. To prevent sudden

change in pruning parameters due to instantaneous change in SNR according to the channel, as in the case of fast fading channels, a minimum updating interval for the parameters can be set in advanced. Such interval may be determined by the channel characteristics.

\section{SIMULATION TESTS AND DISCUSSION}

The three types of polar decoders mainly: SCL, RP-SCL, and ARP-SCL are simulated to determine their complexity and BER performances. The details of the tests, the results and their discussion are presented next.

\section{A. System and Channel Models}

Details of the system and channel models and their parameters are given in the following:

- A polar code with (N, K) of $(256,128)$ is used in the simulation. This code is assisted with the Cyclic Redundancy Code (CRC) for better performance. Block lengths of less than 400 bits are necessary for ultra-reliable low latency (URLL) and massive machine type communication (mMTC) 5G use cases [22]. The three decoders (SCL, RP-SCL, and ARP-SCL) are operated as described in Sections II and III. The list sizes used with the mentioned decoders are 8 and 32.

- The used signal modulation is Quadrature Phase Shift Keying (QPSK) with orthogonal frequency division multiplexing (OFDM) signal format [23]. A carrier frequency in the mmWave band of $28 \mathrm{GHz}$ is used in the model [24]. Two bandwidths of $50 \mathrm{MHz}$ and $400 \mathrm{MHz}$ are utilized giving two arrangements of OFDM with their related cyclic prefix selections[25]. The first arrangement used 512 subcarriers with 36 for cyclic prefix, called here as OFDM1, while the second arrangement used 4096 subcarriers with 288 for cyclic prefix called OFDM2. The mentioned two arrangements OFDM1 and OFDM2 resulted in transmission bit rates of about 90 Mbps and 720 Mbps, respectively [26]. Further, a MIMO system with a dimensionality of $4 \mathrm{x} 4$ using Space-Time Block Coding (STBC) is assumed [27].

- Two-channel models are considered: AWGN channel and fading channel that models 5G environment. The latter channel model is approved by $3 \mathrm{GPP}$ and defined over the frequency range from $0.5 \mathrm{GHz}$ to $100 \mathrm{GHz}$ with a maximum 


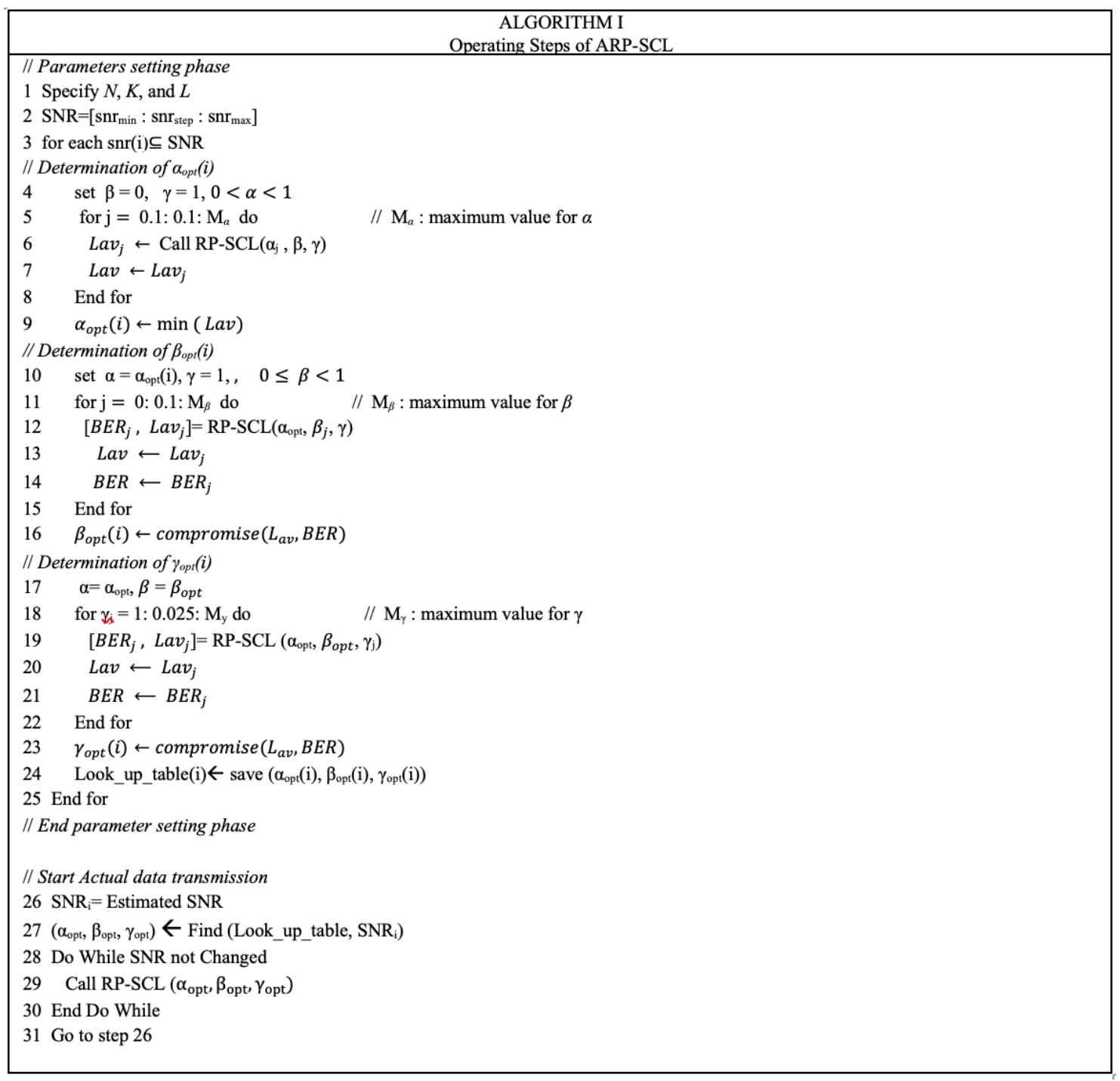

bandwidth of $2 \mathrm{GHz}$. The considered model is known as Type-D channel for indoor office having short-delay profile environment [24]. Tapped delay line channel model with three paths is considered. The path gains are [ - $0.2-18.8$-21]

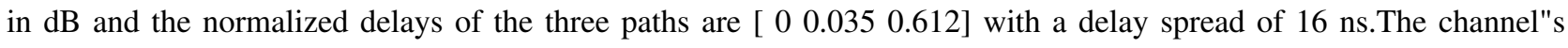
$\mathrm{K}$-factor is taken to be $13.3 \mathrm{~dB}$ [24]. The slow movement is considered, where the relative velocity between the transmitter and the receiver is $3 \mathrm{~km} / \mathrm{Hr}$ resulting in a maximum Doppler shift of $77.884 \mathrm{~Hz}$.Based on the power delay profile and the delay spread, the coherent bandwidth of the considered fading channel is $230 \mathrm{MHz}$ [28].

\section{B. The Calculation of Optimum Pruning Parameters}

To assign different parameter sets of ARP-SCL for each SNR region, the typical BER performance of the SCL decoder is considered to categorize the SNR range for each channel into three regions: low, moderate, and high SNR region. Such 
an approach is being followed here because any SCL based modified decoder should have performance close to that of SCL. According to ARP-SCL operation, the pruning parameters $\left(\alpha_{\mathrm{opt}}, \beta_{\mathrm{opt}}\right.$, and $\left.\gamma_{\mathrm{opt}}\right)$ are determined for different SNR values covering the assumed SNR regions. It is worth to mention here that the SNR regions may vary according to the considered channel model. Table I shows the range of the optimum pruning parameters of ARP-SCL for each channel model for the two lust sizes considered in this work.

TABLE I

CATEGORY OF THE MIT-BIH ECG DATASET

\begin{tabular}{|c|c|c|c|c|c|c|c|c|c|c|c|c|}
\hline \multirow{3}{*}{$\begin{array}{c}\text { Optimum } \\
\text { Pruning }\end{array}$} & \multicolumn{4}{|c|}{ AWGN Channel } & \multicolumn{4}{|c|}{ Fading Channel with $50 \mathrm{MHz}$ Bandwidth using OFDM\#1 } & \multicolumn{4}{|c|}{ Fading Channel with $400 \mathrm{MHz}$ Bandwidth using OFDM\#2 } \\
\hline & \multicolumn{2}{|c|}{$\mathrm{L}=8$} & \multicolumn{2}{|c|}{$\mathrm{L}=32$} & \multicolumn{2}{|c|}{$\mathrm{L}=8$} & \multicolumn{2}{|c|}{$\mathrm{L}=32$} & \multicolumn{2}{|c|}{$\mathrm{L}=8$} & \multicolumn{2}{|c|}{$\mathrm{L}=32$} \\
\hline & Min. & Max. & Min. & Max. & Min. & Max. & Min. & Max. & Min. & Max. & Min. & Max. \\
\hline Parameter & Value & Value & Value & Value & Value & Value & Value & Value & Value & Value & Value & Value \\
\hline$\alpha$ opt & 0.3 & 0.6 & 0.3 & 0.6 & 0.2 & 0.6 & 0.3 & 0.6 & 0.2 & 0.6 & 0.3 & 0.6 \\
\hline$\beta$ opt & 0.24 & 0.487 & 0.117 & 0.36 & 0.159 & 0.32 & 0.173 & 0.39 & 0.19 & 0.4 & 0.161 & 0.318 \\
\hline$\gamma$ opt & 1.042 & 1.156 & 1.047 & 1.1025 & 1.04 & 1.126 & 1.048 & 1.098 & 1.02 & 1.127 & 1.045 & 1.104 \\
\hline
\end{tabular}

The variation in the optimum pruning parameters between their minimum and maximum values is due to the change in SNR. It is clear from Table I, that is unaffected by the channel model and the list size when compared to the other pruning parameters.

\section{The Decoding Complexity}

In general, the indications for the polar decoder complexity are the average processed paths Lav by the decoder, the computational complexity determined by the required mathematical operations in decoding of every codeword, and the memory required by the decoder. The average processed paths $L_{a}$ is usually compared to that required by the standard SCL decoder. The variations of $L_{a v}$ with SNR for SCL, RP-SCL, and ARP-SCL polar code decoder using list sizes of 8 and 32 over AWGN, $50 \mathrm{MHz}$ fading bandwidth, and $400 \mathrm{MHz}$ bandwidth channel are shown in Fig. 1, Fig. 2 , and Fig. 3 , respectively. As shown in these figures, the use of ARP-SCL reduces $L_{a v}$ for most considered SNR regions compared to SCL and RP-SCL decoder. For the AWGN channel, the minimum complexity in term of $L_{a v}$ reduced from about 5.9, and 19.7 paths with RP-SCL to about 5.5 and 18.8 paths with ARP-SCL for list size of 8 and 32, respectively. For the 50 $\mathrm{MHz}$ fading channel case, the corresponding decoder complexity is reduced from about 6.2 and 22 paths with RP-SCL to about 4.7 and 17 paths with ARP-SCL for list size of 8 and 32, respectively. Further, for the $400 \mathrm{MHz}$

fading channel case, the decoder complexity is reduced from about 5.9 and 20 paths with RP-SCL to about 5.2 and 17 paths with ARP-SCL for list size of 8 and 32 respectively.

Tables II and III show the percentage reduction in $L_{a v}$ achieved by RP-SCL and the proposed ARP-SCL decoders as compared to the SCL decoder for list sizes of 8 and 32, respectively, for different SNR regions and channels. The percentage reduction in $L_{a \underline{v}}\left(C_{P R}\right)$ is determined by (6)

$$
C_{P R}=\frac{L-L_{a v}}{L} .100 \%
$$


Both Tables II and III show that the proposed ARP-SCL decoder provided better complexity reduction over the RP-SCL decoder for the different regions of SNR and channels. For $\mathrm{L}=8$ in the high SNR region (Table II), the complexity reduction increased from about $26 \%, 23 \%$, and $26 \%$ for RP-SCL decoder to about $31 \%, 41 \%$, and $35 \%$, for ARP- SCL decoder, for AWGN, $50 \mathrm{MHz}$ bandwidth fading, and $400 \mathrm{MHz}$ bandwidth fading channels, respectively.The corresponding values for $\mathrm{L}=32$ (Table III) is $38 \%, 31 \%$, and $38 \%$ to about $41 \%, 47 \%$, and $47 \%$, respectively.

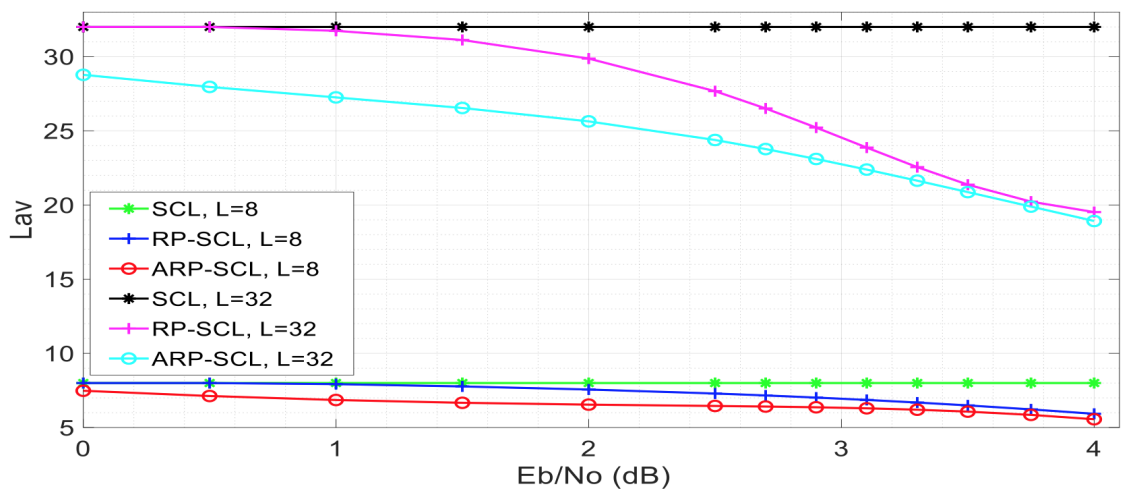

Figure 1: Variations of $L_{a v}$ with SNR of different decoders over AWGN channel.

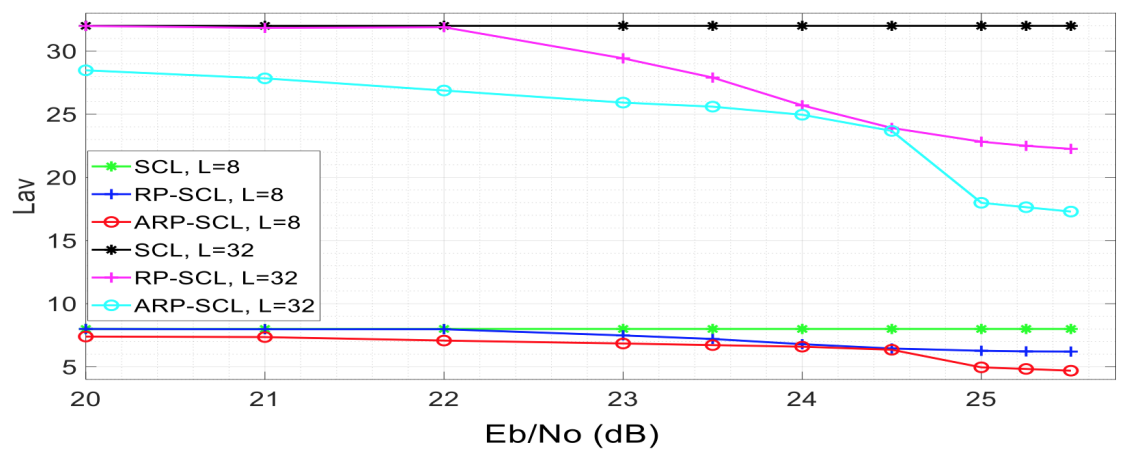

Figure 2: Variations of $L_{a v}$ with SNR of different decoders over mmWave fading channel with $50 \mathrm{MHz}$ bandwidth

TABLE II

PERCENTAGE REDUCTION IN LAV RELATIVE TO SCL DECODER WITH L=8 AT DIFFERENT SNR REGIONS

\begin{tabular}{|c|c|c|c|c|c|c|}
\hline \multirow{2}{*}{ Channel Model } & \multicolumn{2}{|c|}{ Low SNR Region } & \multicolumn{2}{c|}{ Moderate SNR Region } & \multicolumn{2}{c|}{ High SNR Region } \\
\cline { 2 - 7 } & RP-SCL & ARP-SCL & RP-SCL & ARP-SCL & RP-SCL & ARP-SCL \\
\hline AWGN & $1.25 \%$ & $13.75 \%$ & $3.125 \%$ & $16.8 \%$ & $26.25 \%$ & $31.25 \%$ \\
\hline Fading/B=50MHz & 0 & $7.50 \%$ & 0 & $7.50 \%$ & $22.50 \%$ & $41.25 \%$ \\
\hline Fading/B=400MHz & 0 & $11.25 \%$ & 0 & $11.25 \%$ & $26.25 \%$ & $35.00 \%$ \\
\hline
\end{tabular}

For moderate and low SNR regions, the proposed ARP-SCL also provides an improvement in complexity reduction over RP-SCL decoder, for all channels and list sizes as shown in both tables. Amazingly, unlike the RP-SCL decoder, the proposed ARP-SCL decoder provided complexity reductions at both the low and moderate SNR regions. The largest 


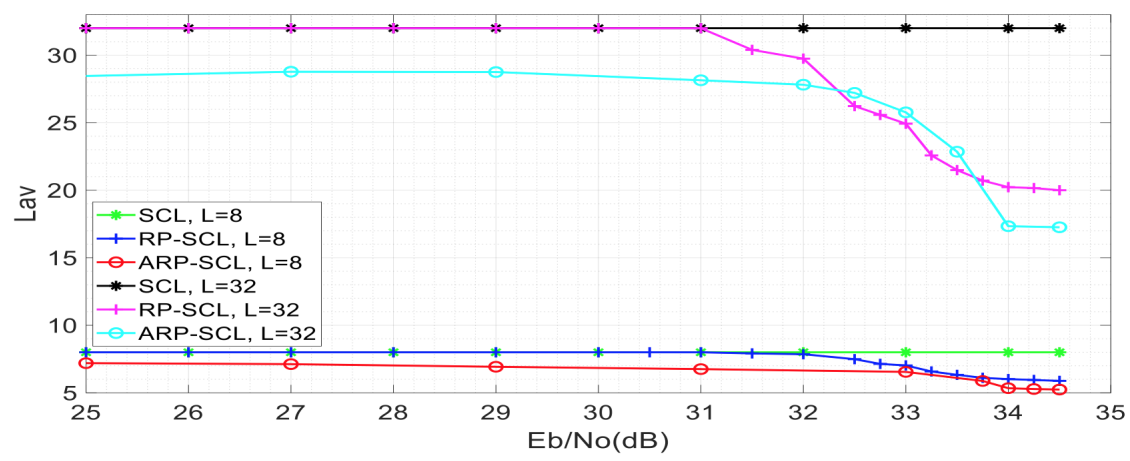

Figure 3: Variations of Lav with SNR of different decoders over mmWave fading channel with $400 \mathrm{MHz}$ bandwidth

TABLE III

PERCENTAGE REDUCTION IN LAV RELATIVE TO SCL DECODER WITH L=32 AT DIFFERENT SNR REGIONS

\begin{tabular}{|c|c|c|c|c|c|c|}
\hline \multirow{2}{*}{ Channel Model } & \multicolumn{2}{|c|}{ Low SNR Region } & \multicolumn{2}{c|}{ Moderate SNR Region } & \multicolumn{2}{c|}{ High SNR Region } \\
\cline { 2 - 7 } & RP-SCL & ARP-SCL & RP-SCL & ARP-SCL & RP-SCL & ARP-SCL \\
\hline AWGN & 0 & $14.8 \%$ & $6.25 \%$ & $20.3 \%$ & $38.44 \%$ & $41.25 \%$ \\
\hline Fading/B=50MHz & 0 & $9.38 \%$ & 0 & $10.94 \%$ & $31.25 \%$ & $46.88 \%$ \\
\hline Fading/B=400MHz & 0 & $12.5 \%$ & 0 & $15.63 \%$ & $37.50 \%$ & $46.66 \%$ \\
\hline
\end{tabular}

such reductions are obtained with the AWGN channel ranging from about $13 \%$ to $20 \%$. For the fading channels these reductions, for the proposed ARP-SCL decoder, ranging from $7.5 \%$ to about $15.6 \%$.

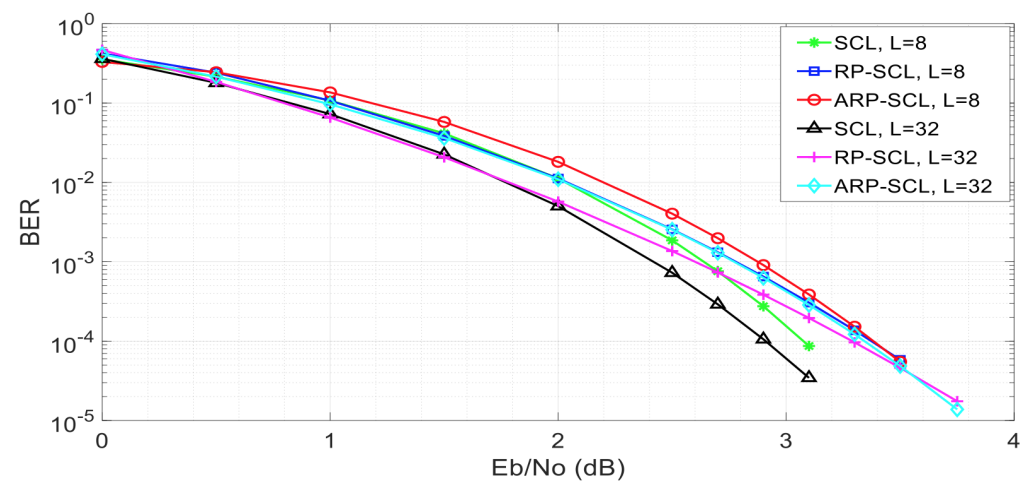

Figure 4: BER performance of decoders over AWGN channel

The reduction in complexity percentage achieved by ARP-SCL at high SNR region is between $31 \%$ and $47 \%$ comparing to SCL decoding. The corresponding reduction achieved at low and moderate SNR regions is from $7.5 \%$ to $20 \%$. This complexity reduction over different SNR regions is related to the effect of SNR on path metric. The comparison shows that such reduction is relatively small at the low SNR region, unlike the high SNR region.

The computations required by the SCL decoder is $\mathrm{O}\left(\mathrm{L} . \mathrm{Nlog}_{2} \mathrm{~N}\right.$ ), while that of RP-SCL decoder is O (Lak- when compared to both RP-SCL and SCL decoders. This is obtained at different SNR regions and for both list sizes and channels.

Considering the required memory in bits for the conventional SCL decoder as given by [14] and used by [19] for 


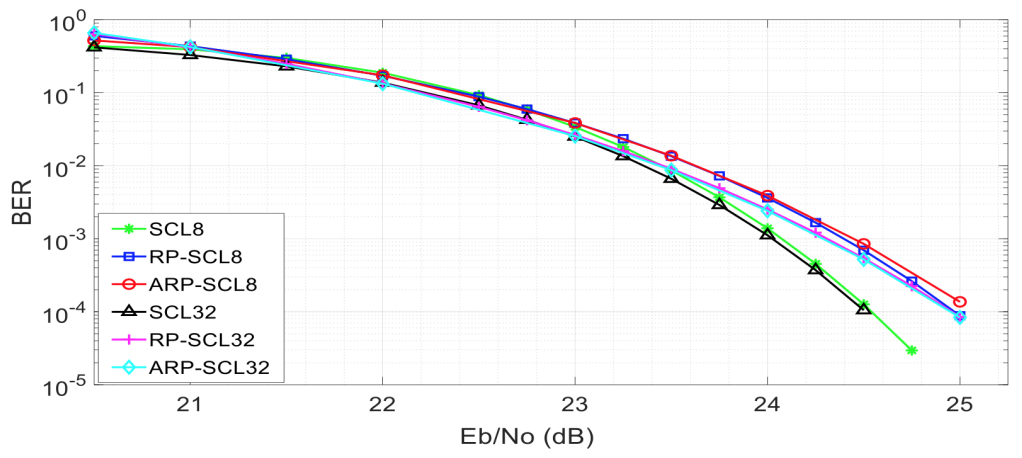

Figure 5: BER performance of different decoders over mmWave fading channel with $50 \mathrm{MHz}$ bandwidth

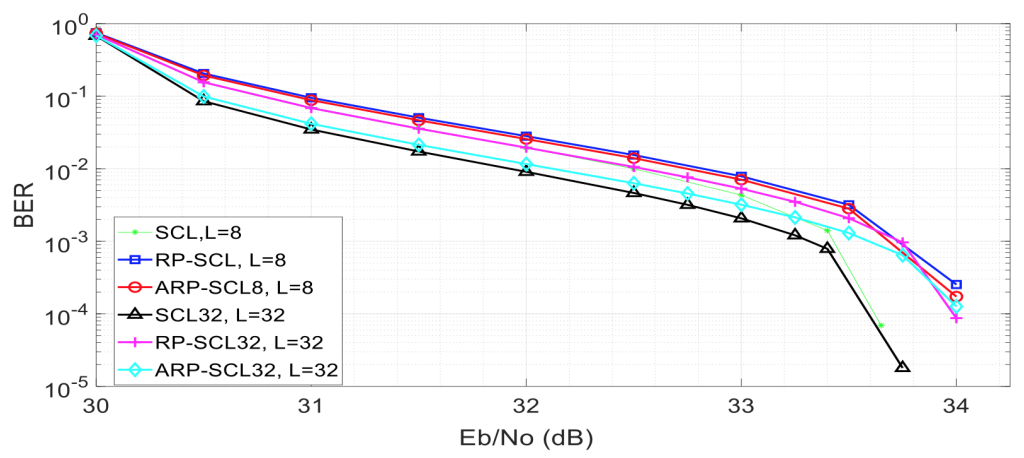

Figure 6: BER performance of different decoders over mmWave fading channel with $400 \mathrm{MHz}$

RP-SCL, one may consider the memory units (in bits) required for the proposed ARP-SCL decoder as in (7):

$$
M_{A R P-S C L}=\left(N+L_{a v-A}(N-1)\right) Q_{L L R}+L_{a v-A} Q_{P M}+L_{a v-A}(2 N-1)
$$

where $Q_{L L R}$ is the number of bits used to quantize the Log-Likelihood Ratio, and $Q_{P M}$ is the number of bits required to quantize the path metrics.

\section{BER Performance}

The BER performance for the considered polar code (PC $(256,128)$ ) using SCL, RP-SCL, and ARP-SCL decoders with the two list sizes of 8 and 32 over AWGN channel, mmWave fading channels with a bandwidth of $50 \mathrm{MHz}$, and $400 \mathrm{MHz}$ are shown in Figs. 4, 5, and 6, respectively. The BER performance of the proposed ARP-SCL decoder is either similar or better than that of the RP-SCL decoder. For mmWave fading channels with a bandwidth of 50, shown in Fig. 5, the performances for both RP-SCL and ARP-SCL are almost identical. On the other hand, for the case of mmWave fading channels with bandwidths of $400 \mathrm{MHz}$ channel, ARP-SCL outperformed RP-SCL. A slight advantage, less than $0.2 \mathrm{~dB}$, is obtained by RP-SCL over ARP-SCL decoder for the case of AWGN channel at moderate SNR region as shown in Fig. 
4. In all test conditions, the SCL BER performance is better than those of RP-SCL and ARP-SCL decoders. This is vital since the SCL decoder did not use any pruning in the decoding process.

\section{E. Discussion of Results}

The above-mentioned decoding complexity (percentage reduction in $L_{a v}$, required computations, and decoder required memory) revealed that the proposed ARP-SCL decoder succeeded in achieving further complexity reduction as compared to the previously suggested RP-SCL decoder at all SNR regions. These improvements are achieved by using different pruning parameter sets for each SNR regions. The additional complexity required by the ARP-SCL decoder for the adaptation is relatively small.This comprises the SNR estimation process, memory required to store the pruning parameter sets, and a simple searching mechanism to find the appropriate set of optimum parameters. In the case of the AWGN channel, percentage reductions in $L_{a v}$ of about $13 \%$ over RP-SCL are obtained for the considered polar code. This is combined with a slight SNR difference (less than $0.2 \mathrm{~dB}$ ) in favor of RP-SCL over ARP-SCL at moderate SNR regions. Over the two mmWave fading channels, the proposed ARP-SCL decoder achieved up to 19\% reductions in Lav over RP-SCL decoder. This complexity reduction is achieved without noticeable degradation in BER performance compared to RP-SCL. Further, in the case of mmWave fading channels with a bandwidth of $400 \mathrm{MHz}$, the BER performance of ARP-SCL is closer to that of the SCL decoder than that of RP-SCL. This indicates that the change of the optimum threshold parameter sets according to the operating SNR region is well suited to the decoding operations of the proposed ARP-SCL in non-ideal channel conditions.

\section{CONCLUSIONS}

An Adaptive Reduced Path Successive Cancellation List (ARP-SCL) decoding algorithm for polar code, proposed in this work, succeed in promoting the percentage of complexity reduction for Successive Cancellation List (SCL) decoder at all Signal to Noise Ratio (SNR) regions for most considered cases compared to the previously proposed Reduced Path Successive Cancellation List (RP-SCL) decoder. The main idea of ARP-SCL is to take the variation in operation SNR into account by considering multiple sets of pruning parameters optimized based on multiple values of design SNR compared to a single set of pruning parameters corresponding to single design SNR used with RP-SCL. The pruning parameter sets of ARP-SCL can be determined before the transmission, so that, no added overhead has resulted during the actual transmission. The performance of ARP-SCL is evaluated over Additive White Gaussian Noise (AWGN) channel and fading channel model with mmWave carrier frequency and two bandwidths of $50 \mathrm{MHz}$

and $400 \mathrm{MHz}$. The results are relevant that the maximum percentage reduction in the average processed paths (Lav) comparing to SCL achieved at high SNR region is increased from about $26 \%$ and $38 \%$ with RP-SCL to about $41 \%$ and $47 \%$ with ARP-SCL over AWGN and fading channels, respectively. The corresponding reduction achieved at low and moderate SNR regions is increased from $6 \%$ and $0 \%$ with RP-SCL to about $20 \%$ and $16 \%$ with ARP-SCL over AWGN and fading channels, respectively. The memory requirement and computational complexity for the ARP-SCL are also reduced with the same percentage of reduction for $L_{a v}$. In most test conditions, the mention reductions in decoder complexity are achieved without sacrificing the BER performance. 


\section{REFERENCES}

[1] E. Arikan, "Channel Polarization: A Method for Constructing Capacity- Achieving Codes for Symmetric Binary- Input Memoryless Channel" , IEEE Trans. Inf. Theory, vol. 55, no. 7, pp. 3051- 3073, July 2009.

[2] W. Abdulwahab, and A. Kadhim, "Internal Pilot Inserton for Polar Codes" , Submitted for publication to Indonesian Journal of Electrical Engineering and Computer Science.

[3] X. Liang,H. Wang, Y. Shen, Z. Zhang, X. You, and C. Zhang, " Efficient Stochastic Successive Cancellation List Decoding for Polar Codes" , Science China Information Sciences vol. 63. no. 10, pp. 202303:1- 202303:19, Oct. 2020.

[4] P. Marsch, Bulakcl, O. Quesetb, and M. Boldi, "5G System Design Architectural and Functional Considerations and Long Term Research" , John Wiley \& Sons Ltd, 2018, ch. 11.

[5] D. Chandramouli, R. Liebhart, and J. Pirskanen, "5G for the Connected World" , John Wiley \& Sons Ltd, 2019, pp:73- 76.

[6] W. Abdulwahab, and A. Kadhim, "Comparative Study of Channel Coding Schemes for 5G" , in International Conference on Advanced Science and Engineering (ICOASE), Duhok, Iraq, Oct. 2018 .

[7] A. Kadhim and A. Hamad, "Turbo Codes with Internal Pilot Insertion", in 2nd International Conference on Next Generation Mobile Applications, Services, and Technologies (NGMAST), Cardiff, U. K, Sept. 2008 .

[8] I. Tal and A. Vardy "List Decoding of Polar Codes" , IEEE Trans. Inf. Theory, vol. 61, no. 5, pp. 2213- 2226, May 2015.

[9] V. Bioglio, C. Condo, I. Land, and S. Member, "Design of Polar Codes in 5G New Radio" , 2018 [Online]. Available: https://arxiv.org/abs/1804.04389.

[10] K. Chen, K. Niu, and J. Lin, "A Reduced- Complexity Successive Cancellation List Decoding of Polar Codes" , in IEEE 77th Vehicular Technology Conference (VTC Spring), Dresden, Germany, June 2013.

[11] A. Balatsoukas- Stimming, M. B. Parizi, and A. Burg, "LLR- Based Successive Cancellation List Decoding of Polar Codes" , IEEE Trans. Signal Process. , vol. 63, no. 19, pp. 5165- 5179, Oct. 2015.

[12] K. Chen, B. Li, H. Shen, J. Jin, and D. Tse, "Reduce The Complexity of List Decoding of Polar Codes by Tree- Pruning" , IEEE Commun. Lett. , vol. 20, no.2, pp. 204- 207, Feb. 2016

[13] S. Hashemi, A. Balatsoukas- Stimming, P. Gjara, C. Thibeayl, and W. Gross W, "Partitioned Successive- Cancellation List Decoding of Polar Codes" , in IEEE International Conference on Acoustics, Speech and Signal Processing (ICASSP), Shanghai, China, March 2016.

[14] S. Hashemi, M. Mondelli, S. Hassani, R. Urbanke, W. Gross, "Partitioned List Decoding of Polar Codes: Analysis and Improvement of Finite Length Performance", in IEEE Global Communications Conference (GLOBECOM), Singapore, Dec. 2017.

[15] S. Hashemi, et al. , "Decoder Partitioning: Towards Practical List Decoding of Polar Codes" , IEEE Transaction on Communication. vol 66, no. 9, pp. 3749- 3759, Sept. 2018

[16] S. Hashemi,C. Condo, and W. Gross, "Simplified Successive- Cancellation List Decoding of Polar Codes" , in IEEE International Symposium on Information Theory (ISIT), Barcelona, Spain, July 2016.

[17] S. Hashemi, C. Condo, and W. Gross, "Fast Simplified Successive- Cancellation List Decoding of Polar Codes" , in IEEE Wireless Communications and Networking Conference Workshops (WCNCW), San Francisco, USA, March 2017.

[18] C. Condo, V. Bioglio, and I. Land, "Generalized Fast Decoding of Polar Codes" , in IEEE Global Communications Conference (GLOBECOM), Abu Dhabi, United Arab Emirates, Dec. 2018.

[19] W. Abdulwahab, and A. Kadhim, "Reduced Paths Successive Cancellation List Decoding for Polar Codes" , Accepted for publication by International Journal of Engineering and Technology Innovation, Oct. , 2020 .

[20] H. Gamage, V. Ranasinghe, N. Rajatheva, and M. Latva- aho, "Low Latency Decoder for Short Blocklength Polar Codes" , in European Conference on Networks and Communications (EuCNC), Croatia, June 2020.

[21] I. Tal and A. Vardy "List Decoding of Polar Codes", IEEE Transactions on Information Theory, vol. 61, no. 5, pp. $2213-2226,2015$.

[22] A. Sharma and M. Salim, "Polar Code: The Channel Code Contender for 5 G Scenarios" , in International Conference on Computer, Communications and Electronics (Comptelix), Jaipur, India, July 2017.

[23] 3GPP TS 38.211 version 15.8 .0 Release 15, "5G, NR, Physical Channels and Modulation", European Telecommunications Standards Institute, July 2018.

[24] 3GPP TR 38.901 version 16.1.0 Release 16, "5G Study on Channel Model for Frequencies from 0.5 to 100GHz" , European Telecommunications Standards Institute, Nov. 2020.

[25] 3GPP TS 38.101-2 version 15.8.0 Release 15, "5G, NR usereEquipment (UE) radio transmission and reception part 2: range 2standalone" , European Telecommunications Standards Institute, Jan. 2020.

[26] A. Goldsmith, "Wireless Communications" , Cambridge Uiniversity Press, New York, USA, 2005, ch. 12.

[27] 3GPP TS 138.141-1 version 15.0.0 Release 15, "5G, NR, Base Station (BS) Conformance Testing Part 1: Conducted Conformance Testing" , European Telecommunications Standards Institute, April 2019.

[28] D. Tse, and P. Viswanath, "Fundimantals of Wirelees Communication" , Cambridge University Press, New2005, ch. 2. 